\title{
Lithium during the AGB evolution in young open clusters of the Large Magellanic Cloud ${ }^{\star}$
}

\author{
C. Maceroni ${ }^{1}$, V. Testa ${ }^{1}$, B. Plez ${ }^{2}$, P. García Lario ${ }^{3}$, and F. D'Antona ${ }^{1}$ \\ 1 INAF, Osservatorio Astronomico di Roma, via Frascati 33, 00040 Monteporzio C. (RM), Italy \\ e-mail: surname@coma.mporzio.astro.it \\ 2 GRAAL, Université Montpellier II, 34095 Montpellier Cedex 5, France \\ 3 ISO Data Centre, Science Operations and Data Systems Division, Research and Scientific Support Department of ESA, \\ VILSPA, 28080 Madrid, Spain
}

Received 29 July 2002 / Accepted 27 August 2002

\begin{abstract}
We present the results of mid-resolution spectroscopy in the Li I $6708 \AA$ spectral region of Asymptotic Giant Branch (AGB) stars belonging to young open clusters of the Large Magellanic Cloud. Most stars belong to the clusters NGC 1866 and NGC 2031, which have an age of $\simeq 150$ Myr. Lithium lines of different strength are detected in the spectra of stars evolving along the AGB, in agreement with theoretical predictions. According to stellar evolutionary models, at the start of the AGB the stars should all show a low residual lithium abundance as a consequence of dilution during the previous evolutionary phases. The most luminous and cooler thermally pulsating AGB stars, if they are massive enough, once in the AGB go first through a phase of Li destruction, which is followed by a phase of strong lithium production and further destruction. The production of lithium on the AGB is in particular explained by the onset of the "Hot Bottom Burning" (HBB) process. Our most conclusive results are obtained for the populous cluster NGC 1866 where: the "early-AGB" stars show a weak Li line, which can be attributed to the dilution of the initial abundance; one of the two luminous stars seem to have completely depleted lithium, as no line is detected; the second one shows a deep lithium line, whose strength can be explained by lithium production. The bolometric magnitude of these stars are consistent with the values predicted by the theory, for the mass evolving on the AGB of NGC 1866, at which lithium is first destroyed and then produced $\left(M_{\mathrm{bol}} \simeq-6\right.$ ). We also analyze the infrared luminosities (ISOCAM data) of these stars, to discuss if their evolutionary phase precedes or follows the lithium production stage. More intriguing and less clear results are obtained for the most luminous stars in NGC 2031: the brightest star seems as well to have destroyed lithium, while the second one shows a strong lithium line. However its bolometric luminosity - derived from the near-IR photometry, is much lower $\left(M_{\text {bol }} \simeq-5.2 \pm 0.15\right)$ than that expected from HBB models. Although low luminosity lithium rich AGB stars are also known, whose appearance is attributed to non-canonical mixing processes, it is not clear why two almost coeval clusters show such a different behaviour. It is also possible that this star does not belong to NGC 2031. Finally we suggest the observational tests that could shed further light on this matter.
\end{abstract}

Key words. stars: abundances - stars: AGB and post-AGB - galaxies: Magellanic Clouds

\section{Introduction}

The evolution of lithium in intermediate mass stars is a powerful probe of the convective envelope conditions. In particular, during the Asymptotic Giant Branch (AGB) phase in stars of mass large enough for the Hot Bottom Burning (HBB) process to occur, the lithium abundance has a complex behaviour, whose knowledge can shed light on the efficiency of convection and on the occurrence of convective overshooting in the preceding phases. The most suitable targets for such a study are the relatively massive and thermally pulsating (TP) AGB

Send offprint requests to: C. Maceroni,

e-mail: maceroni@coma.mporzio.astro.it

* Based on observations collected at the European Southern Observatory, La Silla, Chile. stars in (young) clusters, whose mass can, in principle, be derived by fitting the cluster color-magnitude diagram. The need for a relatively well populated AGB naturally favors the choice of young clusters in the Large Magellanic Cloud.

We carried out, therefore, an observing program to explore the lithium abundance in the TP-AGB phase of stars belonging to four Large Magellanic Cloud open clusters and whose initial mass was determined by fitting the morphology of the color-magnitude diagram (CMD), turnoff included. We obtained mid-resolution spectra of several stars in NGC 1866 and NGC 2031 (which have ages $\simeq 150 \mathrm{Myr}$ ), of one star in the younger $(\simeq 100 \mathrm{Myr}$ old) cluster NGC 2214 and of one star in the older NGC 2107 ( $\simeq 250 \mathrm{Myr})$ (e.g. Corsi et al. 1994; Girardi et al. 1995). The most luminous (three) stars in NGC 1866 and (two) in NGC 2031, and the stars in the other clusters, were 
selected from the list of Frogel et al. (1990, hereafter FMB90). Being these also the latest spectral type clusters' stars, they are good TP-AGBs candidates. Additional stars were selected in NGC 1866 and NGC 2031, as good candidates for the "earlyAGB" phase of evolution on the base of our own near IR photometry.

We looked for and derived the strength of the lithium line at $\lambda=6707.8 \AA$, and explored its dependence on the AGB luminosity and on the cluster age. The observed spectra were compared with synthetic ones, for evaluation of the lithium abundance. Though a higher dispersion is required for a precise abundance determination, our mid-resolution spectra already provide interesting results.

In the next sections we present the theoretical background of our project (Sect. 2), the criteria for cluster and target selection (Sect. 3), the observations and data reduction (Sect. 4), the analysis of the lithium abundance (Sect. 5) and its theoretical implications (Sect. 6), the analysis of the evolutionary stage represented by the selected stars (Sect. 7), and the final discussion and conclusions (Sect. 8).

\section{Theoretical background}

The term "Hot Bottom Burning" (HBB) refers to nuclear processing that can take place at the bottom of the convective envelope of massive Asymptotic Giant Branch (AGB) stars during the TP phase. The observations of these stars in the Magellanic Cloud fields (Smith \& Lambert 1989, 1990; Plez et al. 1993; Smith et al. 1995) reveal that the most luminous AGB stars are lithium rich. That is indeed satisfactorily explained by the theoretical models including HBB.

During the giant phase preceding the AGB, lithium is depleted by convective dilution. Therefore, if we find on the TP-AGB an abundance much higher than the residual from the preceding phases, we can safely conclude that lithium is manufactured "in situ". Both the computations performed by considering only envelope models (Sackmann et al. 1974) and those of complete evolutionary models (Sackmann \& Boothroyd 1992) show that a temporary enhancement of the lithium abundance in the atmosphere - in agreement with the previously mentioned observations - can be achieved through the chain ${ }^{3} \mathrm{He}+{ }^{4} \mathrm{He} \rightarrow{ }^{7} \mathrm{Be} \rightarrow{ }^{7} \mathrm{Li}$, provided that the mixing is a noninstantaneous process (Cameron \& Fowler 1971). The occurrence of nuclear burning at the bottom of the convective envelope is exemplified in Fig. 1, which shows the time evolution of luminosity and lithium abundance for a $4 M_{\odot}$ stellar model ascending the AGB (from Ventura et al. 1999). The model has helium and metal mass fraction, respectively, $Y=0.26$ and $Z=0.01$.

The lithium evolution in the AGB can be sketched as a sequence of four different phases: i) at the beginning of the AGB, the stellar photosphere has a residual lithium content that is a remnant from a previous convective dilution phase; ii) lithium is totally destroyed when the temperature at the bottom of the envelope increases above $10^{7} \mathrm{~K}$; iii) later on, production via the Cameron - Fowler mechanism proceeds and a high lithium abundance is reached; iv) finally, after $\simeq 10^{5} \mathrm{yr}$, lithium is depleted again, as a consequence of the exhaustion of ${ }^{3} \mathrm{He}$ in the

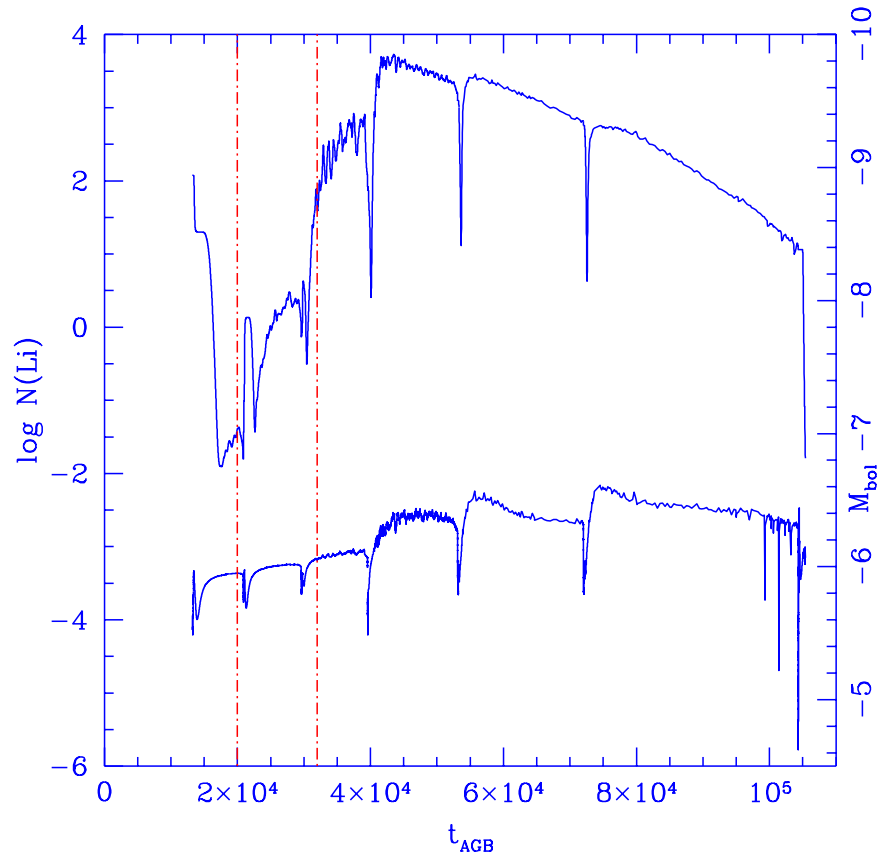

Fig. 1. The evolution of the bolometric magnitude (bottom curve, scale on the right) and of the lithium abundance (top curve, scale on the left) during the TP phase for a $4 M_{\odot}$ with $Z=0.01$ (Ventura et al. 1999). The vertical lines indicate the possible evolutionary phase of the two most luminous AGB stars in NGC 1866.

convective envelope. The stellar mass is, at this stage, $1.7 M_{\odot}$, its mass loss rate is $\simeq 2 \times 10^{-5} M_{\odot} \mathrm{yr}^{-1}$, and the carbon oxygen core mass is $0.82 M_{\odot}$. The evolution will last about $5 \times 10^{4} \mathrm{yr}$ longer, until the whole envelope is lost and the star evolves to the white dwarf stage.

The determination of ${ }^{7} \mathrm{Li}$ abundance along the AGB provides information about the physical processes influencing the efficiency and the extent of mixing, and can help to validate the physical modeling of these processes in stellar interiors.

\section{Clusters and target selection}

The aim of our observations was to understand the correlation between the mass of the stars evolving along the AGB and the lithium abundance. This cannot be done on field giants, because their masses are unknown, while the age of clusters (and therefore the masses of the stars evolving along the AGB) can be derived from the turnoff and/or from the location of the helium core burning (clump) stars. We mainly used for this purpose the recent models by Ventura et al., in preparation, described in Kalirai et al. (2001). For the same models, the evolution is completed through the HBB phases as described in Ventura et al. (2000).

Unfortunately there are not many AGB stars in the young LMC open clusters and it is not well known which ones, among the few M type giants in the clusters, are in the TP phase. Our sample of targets was assembled including the candidates from the list of FMB90 adding a few objects in the clusters NGC 1866 and NGC 2031 that, from our own near IR photometry (Testa, unpublished), come out to be "early AGB" candidates (see Fig. 2). 


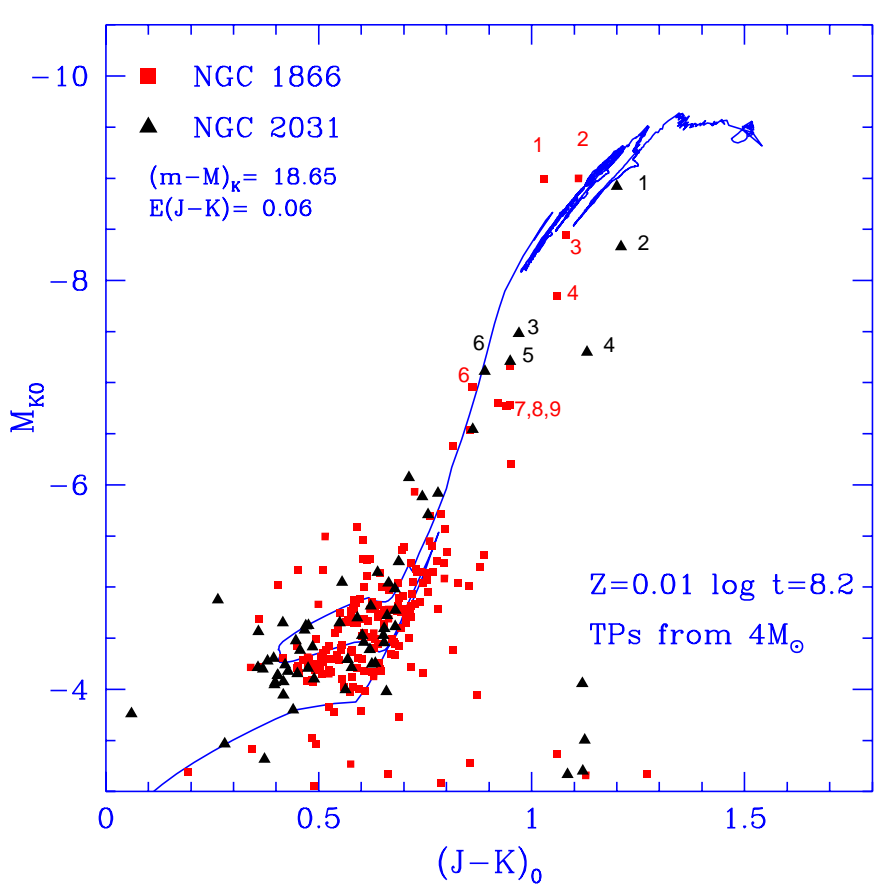

Fig. 2. De-reddened CM diagram of NGC 1866 (squares) and NGC 2031 (triangles). The target stars are identified according to the numbering of Table 1. An isochrone is also shown (from Ventura et al., in prep.) corresponding to a mass in AGB of $\sim 4.3 M_{\odot}$, together with the TP AGB evolution of a $4 M_{\odot}$ star (the difference in mass with respect to the isochrone is of no relevance here). The transformation from the theoretical to the observational diagram was performed by means of the relations of BCP98.

\subsection{Cluster selection}

\subsubsection{NGC 1866}

This cluster, one of the most populous of the LMC, has been very much debated in the literature, as it turns out to be a good test of the possible occurrence of core overshooting during hydrogen burning (see the latest discussion by Barmina et al. 2002). Testa et al. (1999) carefully compared the optical data with models, deriving ages which vary according to the input evolutionary tracks. The authors used either "standard" models, or models with an "extended core" during the hydrogen burning stage, that is, an artificially enlarged convective core (without a physical reason for this extension). This simplified treatment provides limits for the evolving masses which range from $\sim 4.4-5 M_{\odot}$ for the standard case, to $3.9 M_{\odot}$ for the extended core computations (A. Chieffi, private communication). We can compare these results with the models by Ventura et al., in preparation. These include a more physical description of core overshooting, by considering non-instantaneous mixing in the whole core, and extending it beyond the formal convective region. The mixing velocities of these models are taken from the convective model of Canuto et al. (1996). In the overshooting region, the velocities are extrapolated by assuming an exponential decay (consistent with large eddy numerical simulations of convection). The decay scale length is calibrated by means of several observational parameters (first of all, the main sequence observational width), through a parameter $\zeta$ (the larger the value of $\zeta$, the slower the decay). Models with $\zeta=0.02-0.03$ reproduce the observations (Ventura et al. 1998). The same overshooting is also used for the helium-core burning phases. These new models roughly confirm the Testa et al. (1999) computations. The turnoff location and the luminosity level of the red clump stars lead us to date the cluster at ages $\log t=8.05 \div 8.25$ : the smaller value refers to models without overshooting $(\zeta=0)$, the larger one to those with the largest overshooting $(\zeta=0.03)$, and to a composition $Y=0.26$ and $Z=0.006 \div 0.01$. The corresponding evolving mass in the AGB ranges from $4.3(\zeta=0.03)$ to $5 M_{\odot}(\zeta=0)$.

It has to be stressed that the great difference in the evolving masses between scenarios with and without overshooting does not correspond to a large difference in the AGB evolution, as the carbon oxygen core mass, that mainly determines the AGB evolution, is about the same in both cases. Both the $4.3 M_{\odot}$ $(\zeta=0.03)$ and the $5 M_{\odot}(\zeta=0)$ models with $Z=0.01$ will evolve through a HBB phase and produce lithium, at least if the Full Spectrum of Turbulence (FST) efficient convection model Ventura et al. (2000) is adopted.

\subsubsection{NGC 2031}

The CMD of this cluster looks very much similar to that of NGC 1866, although it is much less populated. Because of its location, in a highly populated region of the LMC, the analysis of the photometric data is rather difficult. This, together with the absence of dynamical studies, leaves open the question of the cluster membership of some of its stars. Mould et al. (1993) estimated ages of $140 \pm 20 \mathrm{Myr}$ by means of an isochrone fitting to the optical CMD, and stressed the similarity with NGC 1866.

\subsubsection{NGC 2214 and NGC 2107 : Two comparison clusters}

These two clusters have been selected for comparison with the NGC 1866 and NGC 2031, the two main clusters analyzed in this paper. The first one is definitely younger as can be seen by looking at the CMD published by Banks et al. (1995) or at the one previously available from Robertson (1974). The age estimate for NGC 2214 ranges from 32 Myr (Elson 1991) to $\sim 100 \mathrm{Myr}$ (Girardi et al. 1995). We have re-calibrated the cluster age by using synthetic CMDs built with a grid of models from Ventura et al. (2000), obtaining $t \sim 100$ Myr. NGC 2107 is, on the other hand, older than NGC 1866 and NGC 2031, as found by Corsi et al. (1994) with a $(V, B-V)$ CMD. Girardi et al. (1995) suggest an age of $250 \mathrm{Myr}$ for this cluster. Our recalibration, by using the same method applied for NGC 2214, is in agreement with the above value.

\subsection{Target selection}

The final sample of target stars is listed in Table 1. Each star is identified with the cluster name and is labeled in order of increasing $K$ magnitude. Table 1 lists the magnitudes in various bands. The $B, V$ photometry of NGC 1866 is from Testa et al. (1999); the $R$ photometry for all clusters but NGC 2107 was 
Table 1. Photometric and spectroscopic observations.

\begin{tabular}{|c|c|c|c|c|c|c|c|c|c|}
\hline Star & $J_{0}$ & $H_{0}$ & $K_{0}$ & $R_{0}$ & $V_{0}$ & $B_{0}$ & $N_{\text {obs }}$ & $S / N$ & $\overline{\bar{V}}_{\text {helio }}$ \\
\hline N1866\#1 & 10.70 & 9.89 & 9.67 & 13.81 & 15.38 & 17.06 & 3 & $42,37,40$ & $305 \pm 5$ \\
\hline$N 1866 \# 2$ & 10.77 & 9.91 & 9.66 & 13.80 & 14.97 & 16.61 & 2 & 48,55 & $304 \pm 5$ \\
\hline N1866\#3 & 11.29 & 10.44 & 10.21 & 14.14 & 15.67 & 17.24 & 2 & 39,33 & $306 \pm 6$ \\
\hline N1866\#4 & 11.87 & 11.01 & 10.81 & 13.72 & 14.84 & 16.52 & 1 & 42 & $307 \pm 2$ \\
\hline N1866\#5 & 12.45 & 11.66 & 11.50 & 13.82 & 14.86 & 16.43 & 1 & 65 & $298 \pm 1$ \\
\hline N1866\#6 & 12.56 & 11.79 & 11.70 & 13.85 & 14.75 & 16.21 & 1 & 57 & $298 \pm 3.4$ \\
\hline N1866\#7 & 12.78 & 12.03 & 11.86 & 14.11 & 14.91 & 16.34 & 1 & 52 & $299 \pm 2.7$ \\
\hline N1866\#8 & 12.83 & 12.04 & 11.88 & 14.21 & 15.23 & 16.74 & 1 & 30 & $300 \pm 2.5$ \\
\hline N1866\#9 & 12.83 & 12.07 & 11.89 & 14.12 & 15.14 & 16.58 & 1 & 12 & $307 \pm 7$ \\
\hline N2031\#1 & 10.93 & 10.12 & 9.73 & 14.61 & & & 2 & 52,60 & $235 \pm 6$ \\
\hline N2031\#2 & 11.53 & 10.62 & 10.32 & 13.66 & & & 2 & 21,22 & $259 \pm 6$ \\
\hline N2031\#3 & 12.14 & 11.33 & 11.17 & 13.54 & & & 2 & 53,66 & $248 \pm 2.2$ \\
\hline N2031\#4 & 12.48 & 11.64 & 11.35 & 15.08 & & & 0 & - & - \\
\hline N2031\#5 & 12.39 & 11.59 & 11.44 & 13.81 & & & 1 & 57 & $225 \pm 2$ \\
\hline N2031\#6 & 12.43 & 11.64 & 11.54 & 14.91 & & & 1 & 40 & $237 \pm 8$ \\
\hline$N 2214 \# 1^{a}$ & 10.88 & 10.12 & 9.90 & & & & 1 & 56 & $260 \pm 3$ \\
\hline$N 2107 \# 1^{a}$ & 10.91 & 10.07 & 9.79 & & & & 1 & 42 & $272 \pm 7$ \\
\hline
\end{tabular}

${ }^{a}$ The magnitudes of NGC 2214 and NGC 2107 are not de-reddened.

obtained from calibration shots taken in November 1999 with SUSI2 - NTT; the $J, H, K$ magnitudes of NGC 1866 and 2031 were obtained in 1995 at ESO $2.2 \mathrm{~m}$ - IRAC2. However, for the three brightest stars of NGC 1866 we had to adopt the $J, H$, $K$ magnitudes by FMB90. This choice was preferred because these bright objects fall in the non-linear regime of the infrared IRAC2 detector. The $B$ and $V$ magnitudes of NGC 2214 are from the original work of Robertson (1974).

In order to de-redden the magnitudes of NGC 1866 we adopted $A_{J}=0.09, A_{H}=0.06, A_{K}=0.03$ and $A_{V}=0.25$. The absorption in the $R$ and $B$ bands were derived by means of the Rieke \& Lebofsky (1985) relations for absorption in different bands. The reddening of NGC 2031 is more uncertain: Mould et al. (1993) gives $E(B-V)$ spanning from 0.06 to 0.18 . On the basis of the good match of the red clump of the two clusters we decided to adopt the same reddening values for both of them.

Figure 2 shows the near IR color magnitude diagram for NGC 1866 and NGC 2031 (Testa, unpublished), from which most of the targets have been selected, and a superposed isochrone by Ventura et al., in preparation, describing the clump stars and the early AGB evolution. An age of $\log t=8.2$ was chosen on the basis of the fit of the whole CM diagram ( $V$ versus $B-V$ from Testa et al. 1999), including the turnoff.

The TP AGB phase is sketched on the same plot, by adopting an evolutionary mass of $4 M_{\odot},(Y=0.26, Z=0.01)$ from Ventura et al. (2000). The track includes mass loss according to Blöcker (1995) formulation, with the Reimer's parameter $\eta$ fixed at 0.01 .
Only one star per cluster could be selected in NGC 2214 and NGC 2107 from the sample of FMB90, namely star B69 of NGC 2214 (the notation refers to the work of Robertson 1974), and star No. 6 of NGC 2107.

\section{Observations and data reduction}

The target stars were observed during two nights, in December 1999, with the ESO New Technology Telescope (NTT) and EMMI in the red mid-resolution spectrographic mode. The selected grating (\#4) has a nominal resolution of 5500 (for the $1^{\prime \prime}$-wide slit we used), however the seeing conditions of one night were so good $\left(0.5^{\prime \prime}-0.6^{\prime \prime}\right)$ that for a part of the spectra the actual resolution is actually higher than the nominal value. A total of 23 long slit spectra were obtained for 16 out of the 17 stars in the initial sample, all of them are $640 \AA$ long and are centered on the Li I $6707.8 \AA$ line; the details are given in the last column of Table 1. Some of the stars of NGC 1866 and NGC 2031 were observed several times. The number of spectra available for each target is listed in Col. 7 of Table 1 together with the mean $S / N$ ratio of the individual spectra. Note that star \#4 of NGC 2031, though present in the initial target list, was not observed because of its rather low priority on the basis of its blue color and uncertain classification, however to avoid confusion with the data file names we kept the initial numbering of the sample. The data reduction was performed by means of standard techniques (the long slit reduction 


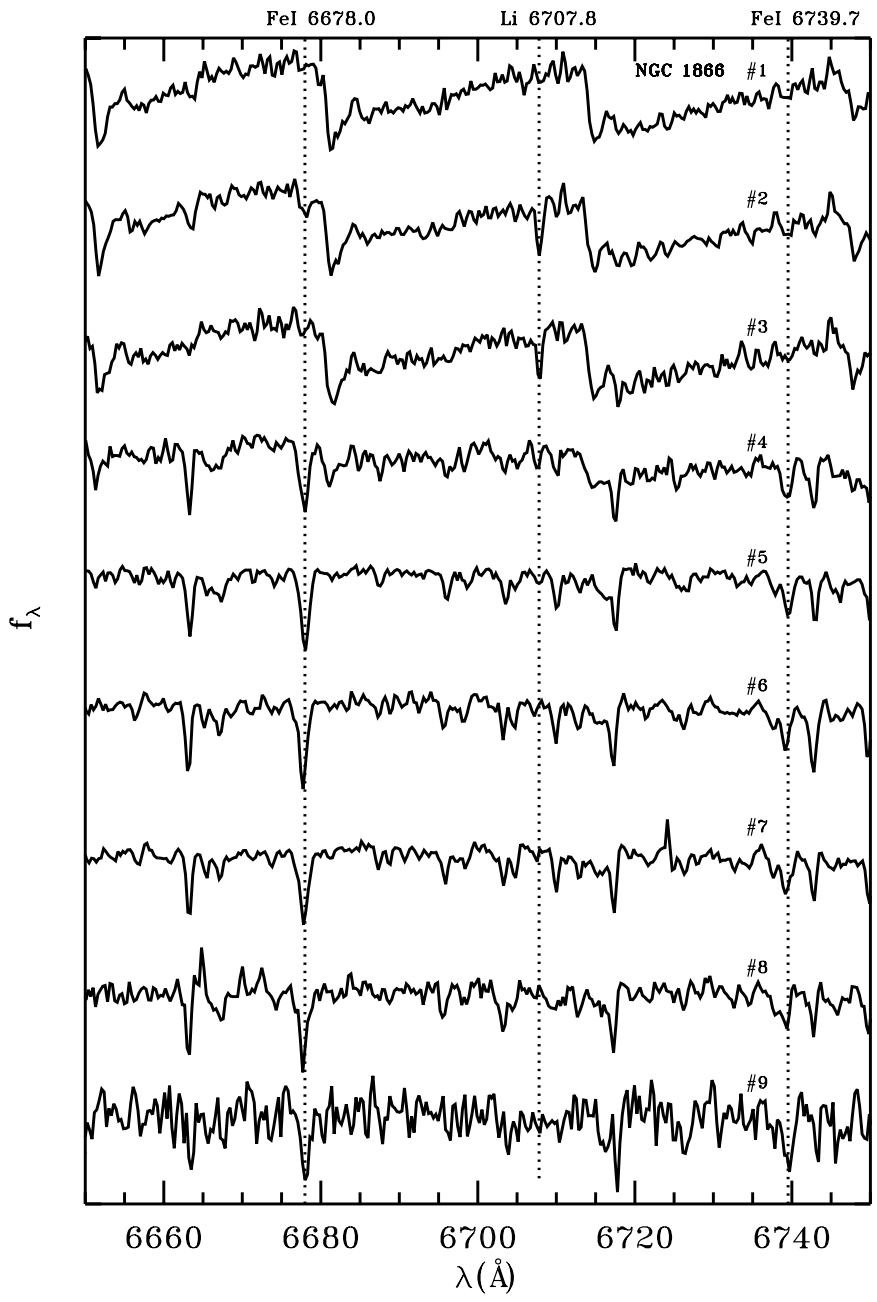

Fig. 3. The spectra in the Li I $6707.8 \AA$ region of the nine AGB stars observed in NGC 1866. The stars are ordered from top to bottom by decreasing near-IR luminosity.

routines of the IRAF ${ }^{1}$ package). The steps were bias subtraction, flat fielding, (partial) cosmic ray removal, normalization, wavelength calibration. The Doppler shifts were derived from $\mathrm{H} \alpha$ and a few other reference lines (mainly Fe I), and the corresponding radial velocities (or a mean value when more spectra were available) are given in the last column of Table 1 . This simple derivation provides relatively large errors, but the results are at least consistent with those from more accurate estimations: for instance, the Doppler shifts derived for the whole sample of nine NGC 1866 AGB stars, yield a mean velocity $V=302.7 \pm 3.6 \mathrm{~km} \mathrm{~s}^{-1}$, in good agreement with previous dynamic studies: according to Fischer et al. (1992) the systemic velocity of NGC 1866 is $301 \pm 1.2 \mathrm{~km} \mathrm{~s}^{-1}$. The small deviations of the individual stars from the mean value confirm as well their cluster membership.

Figures 3 and 4 show the reduced spectra normalized to the maximum flux in the wavelength range displayed.

${ }^{1}$ IRAF is distributed by the National Optical Astronomical Observatories, which are operated by the Association of the Universities for Research in Astronomy, Inc., under cooperative agreement with the National Science Foundation.

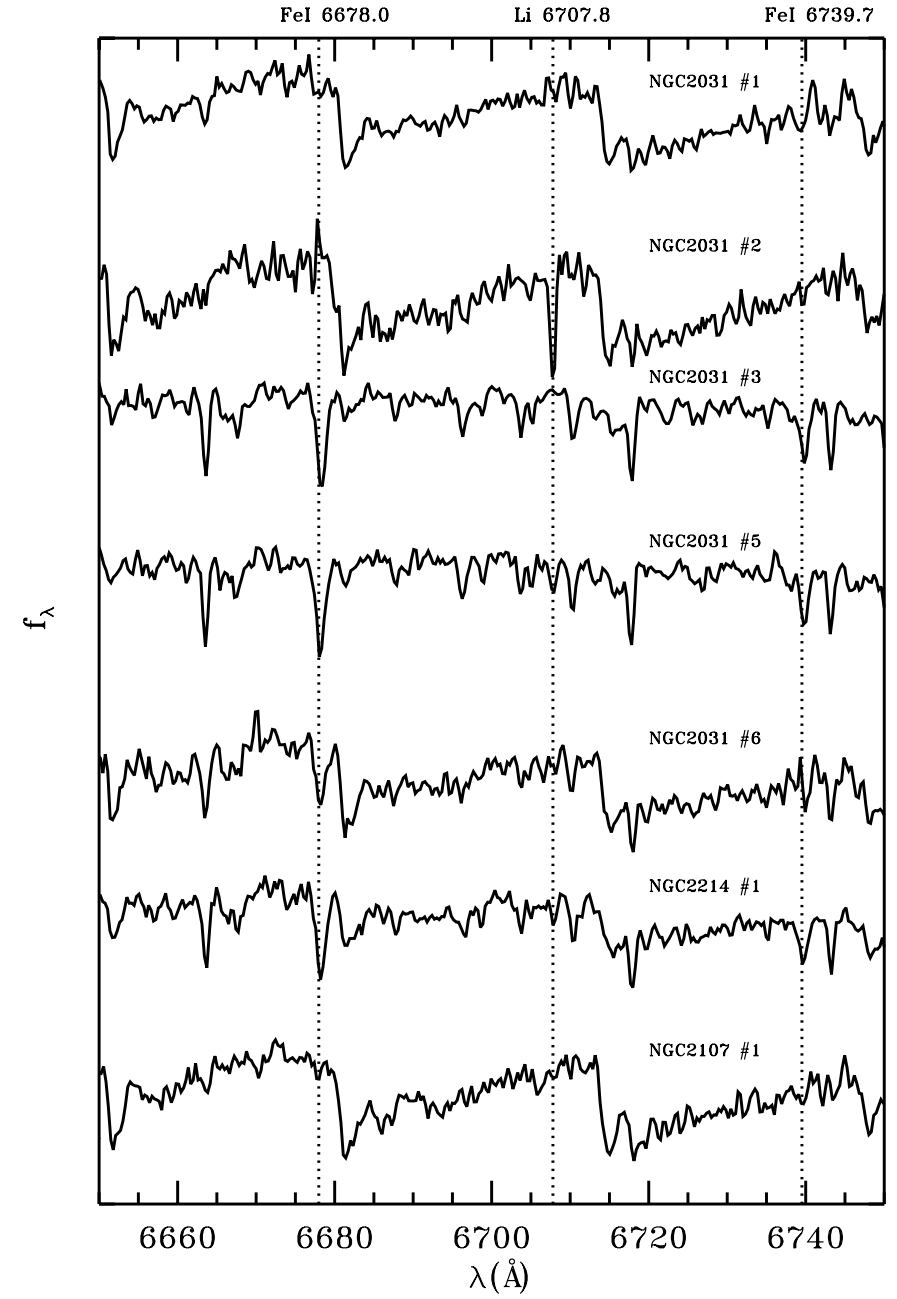

Fig. 4. The spectra in the Li I 6707.8 $\AA$ region of the seven AGB stars observed in NGC 2031, together with those of the AGB stars observed in the comparison clusters NGC 2214 and NGC 2107.

A Li I line at $6708 \AA$ of decreasing strength is clearly visible in stars \#2, \#3, \#4 of NGC 1866, but is absent in the $K$-band brightest star \#1. The same seems to happen in NGC 2031, where again the spectrum of star \#2 shows a strong Li I line, while that of star\#1 one does not. Figure 5 shows a comparison between the observed spectrum of NGC 1866 and two synthetic spectra with reasonable $T_{\text {eff }}$ and lithium content, where it is evident that the lithium abundance of this star must be large. A detailed analysis of the lithium abundances of each star is presented in the next section.

In addition, three different images of the NGC 1866 field obtained with ISOCAM, the mid-IR camera onboard the Infrared Space Observatory (ISO), were retrieved from the ISO Data Archive (TDT numbers: 59000438,59000439 and 59000440) in order to study the mid-IR emission of the AGB sources under analysis in this paper and search for other possible heavily obscured AGB stars in the same field not listed in Table 1. The images were taken through the broadband ISOCAM LW1, LW2 and LW10 filters, whose passbands are centered at 4.5, 6.7 and $12.0 \mu \mathrm{m}$ respectively (Blommaert et al. 2002). They were originally part of the proposal TTANABE.REDSTAR. They were all taken on 


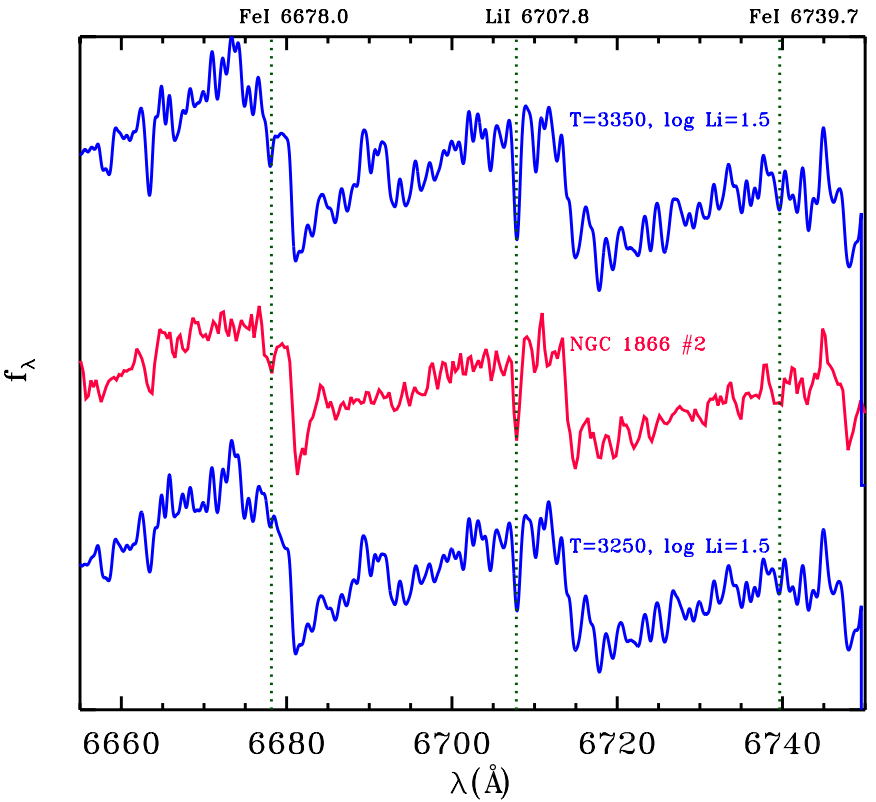

Fig. 5. Comparison among the spectrum of NGC $1866 \# 2$ and two synthetic spectra computed for $T_{\text {eff }}=3250,3350 \mathrm{~K}, \log N(\mathrm{Li})=1.5$, $\log g=0.0$ and $[\mathrm{Fe} / \mathrm{H}]=-0.4$.

28 June 1997 and cover a region of $192^{\prime \prime} \times 192^{\prime \prime}$ with a pixel scale of $3.0^{\prime \prime} \mathrm{pix}^{-1}$.

Data reduction was performed using the "CAM Interactive Analysis" (CIA, version 4.0) starting from the raw data products which were corrected for dark current, glitches, transients and flat-field, following the standard routines available within CIA. An accuracy better than $20 \%$ is expected even at these very low flux levels in the absolute flux assigned to the end products.

\section{Determination of the lithium abundances}

The analysis was performed by comparing our observations with a grid of synthetic spectra with different temperatures and lithium abundances.

The synthetic spectra were calculated with the Turbospectrum program (Alvarez \& Plez 1998), using MARCS model atmospheres (Gustafsson et al. 1975; Plez et al. 1992; Asplund et al. 1997; Gustafsson et al. 2003). The models and the spectra are spherically symmetric, at LTE, and include up-to-date continuous and line opacities for a large number of atoms and molecules. Some details are provided in Hill et al. (2002). The line lists for $\mathrm{VO}, \mathrm{ZrO}, \mathrm{CN}$ and $\mathrm{TiO}$ (Plez 1998), and atomic lines (VALD, Kupka et al. 1999) were used. The spectra were initially computed at a resolution of $0.01 \AA$, and then degraded to match the resolution of the observations.

The stellar parameters needed for synthetic spectra computation $\left(T_{\text {eff }}, \log g\right)$ were initially derived from the photometry. The $K$ band bolometric correction $B C_{K}$ was obtained by using the $B C_{K}$ versus $V-K$ relation by (Bessell et al. 1998, BCP98) for the nine stars of NGC 1866 that have $V-K$ colors. The same stars were used to derive a $B C_{K}$ versus $J-K$ relation that was needed to derive the bolometric correction for the stars of the other clusters, for which $V-K$ is not available.
The bolometric magnitudes were computed assuming a distance modulus for the LMC of $m-M=18.6$. The $T_{\text {eff }}$ values were obtained by means of the $T_{\text {eff }}$ versus $J-K$ and $T_{\text {eff }}$ vs. $V-K$ relations by BCP98, and these, together with $M_{\text {bol }}$ and an assumed mass of $4.5 M_{\odot}$ finally yield the gravity values. A small grid of synthetic spectra with various lithium abundances was generated for the derived parameters. We found that the photometric $T_{\text {eff }}$ did not allow a good fit of the spectra of the cooler stars. Therefore, in order to derive the abundances, new values of $T_{\text {eff }}$ were set by looking at the best overall fit over the 6350-7100 ̊ region. AGB-tip stars are known to be long-period variables, thus we expect differences between the $T_{\text {eff }}$ derived from photometry and the $T_{\text {eff }}$ used for spectroscopy (photometric and spectroscopic observations were not simultaneous).

Moreover, we do not expect the above mentioned transformations to be very accurate for AGB-TP stars. Therefore, in the following discussion, we will stick to the spectroscopic $T_{\text {eff }}$ values. We will use, for instance, the $M_{\text {bol }}$ values that can be derived using backwards the spectroscopic $T_{\text {eff }}$ and the abovementioned calibrations. The results are listed in Table 2.

\section{Comparison with theoretical models}

\subsection{NGC 1866}

Despite the rather large uncertainties on the derived $\mathrm{Li}$ abundances, we have a number of interesting results.

Starting from the bottom of the AGB, we can conclude that the early AGB stars in NGC 1866 do show lithium, although the derived abundance is very $\operatorname{low}(\log N(\mathrm{Li}) \simeq 0$ ). This is consistent with the fact that for a large progenitor mass, we do not expect lithium to be destroyed in the evolutionary phases preceding AGB but only to be diluted, when the convective envelope sinks into the star. Figure 1 shows, however, that the residual abundance of our models (for a $4 M_{\odot}$ ) is $\log N(\mathrm{Li}) \sim 2.0$, i.e. much larger than the observed one. It has to be noticed that these models have a solar-type initial abundance $(\log N(\mathrm{Li}) \simeq 3.3)$, which by comparison with the early AGB value, implies a dilution factor $\sim 20$. If the initial abundance were somewhat lower, say $\log N(\mathrm{Li}) \simeq 2.7$, the diluted abundance would be accordingly scaled. That, however, cannot fully explain the discrepancy: it is highly plausible that an additional mixing mechanism providing further dilution is at work. The occurrence of such a mechanism is well established, at least for lower mass red giants, by the low ${ }^{12} \mathrm{C} /{ }^{13} \mathrm{C}$ and low lithium found on the red giant branch (e.g. Charbonnel 1995). We could also suggest that the lower abundances are due to a mass loss larger than that included in the Ventura et al. (2000) models. Anyway, the models can still be used for comparison, as the memory of the initial value is lost after the first lithium dilution phase.

In spite of this quantitative discrepancy, the presence of lithium in the early AGB stars proves that in this phase the star preserves some lithium, as expected from theory. The models do not predict any lithium production here, so we regard this result as a firm point: prior to the AGB, lithium is present but heavily diluted, and its abundance in the envelope sets an upper 
Table 2. Observed and derived target properties.

\begin{tabular}{|c|c|c|c|c|c|c|c|c|c|}
\hline ID & $M_{\mathrm{k}}$ & $B C_{K}$ & $M_{\text {bol }}$ & $\begin{array}{r}\log T_{\text {eff }} \\
\text { from }\end{array}$ & $\begin{array}{l}\log g \\
\text { phot. }\end{array}$ & $\begin{array}{l}\log T_{\text {eff }} \\
\text { adopt. }\end{array}$ & $\begin{array}{r}\log g \\
\text { values }\end{array}$ & $\log N(\mathrm{Li})$ & Comment \\
\hline N1866\#1 & -8.93 & 2.94 & -5.99 & 3697. & 0.01 & 3350 & 0.0 & $<-0.5$ & ISO detected \\
\hline N1866\#2 & -8.94 & 2.90 & -6.04 & 3529. & -0.09 & 3250 & 0.0 & $1.5 \pm 0.5$ & ISO detected \\
\hline N1866\#3 & -8.39 & 2.91 & -5.48 & 3592. & 0.16 & 3450 & 0.2 & $0.5 \pm 0.5$ & ISO detected \\
\hline N1866\#4 & -7.79 & 2.68 & -5.11 & 3634. & 0.33 & 3750 & 0.5 & $-0.25 \pm 0.3$ & \\
\hline N1866\#5 & -7.10 & 2.52 & -4.58 & 3866. & 0.65 & 3950 & 0.8 & $-0.25 \pm 0.3$ & \\
\hline N1866\#6 & -6.90 & 2.45 & -4.45 & 4055. & 0.78 & 4100 & 0.9 & $+0.0 \pm 0.3$ & \\
\hline N1866\#7 & -6.74 & 2.45 & -4.29 & 3929. & 0.79 & 4000 & 0.9 & $-0.25 \pm 0.3$ & \\
\hline N1866\#8 & -6.72 & 2.52 & -4.20 & 3866. & 0.80 & 3950 & 0.8 & $+0.0 \pm 0.25$ & \\
\hline N1866\#9 & -6.71 & 2.50 & -4.21 & 3887. & 0.81 & 4000 & 0.9 & $<0.5(?)$ & very noisy \\
\hline N2031\#1 & -8.87 & 3.06 & -5.81 & 3340. & -0.10 & 3450 & 0.0 & $<-0.5$ & \\
\hline N2031\#2 & -8.28 & 3.08 & -5.20 & 3319. & 0.14 & 3350 & 0.0 & $2.5 \pm 0.5$ & \\
\hline N2031\#3 & -7.43 & 2.59 & -4.84 & 3824. & 0.53 & 3950 & 0.8 & $<-0.5$ & \\
\hline N2031\#4 & -7.25 & 2.92 & -4.33 & 3487. & 0.57 & & & & not observed \\
\hline N2031\#5 & -7.16 & 2.55 & -4.61 & 3866. & 0.64 & 3950 & 0.8 & $0.2 \pm 0.2$ & \\
\hline N2031\#6 & -7.06 & 2.42 & -4.64 & 3992. & 0.68 & 3450 & 0.2 & $<-0.5$ & \\
\hline N2214\#1 & -8.70 & 2.61 & -6.09 & 3802. & 0.02 & 3650 & 0.3 & $-0.5 \pm 0.3$ & \\
\hline N2107\#1 & -8.81 & 2.90 & -5.91 & 3508. & -0.05 & 3450 & 0.2 & $<-0.5$ & \\
\hline
\end{tabular}

limit to its content during the following evolutionary phases, in absence of further production.

The spectrum of the cooler star N1866\#3 shows the TiO bands and a relatively strong lithium line. However, this star is not yet as luminous as we would expect for lithium production by HBB. It has to be noticed that because of the somewhat lower quality of the spectra, the uncertainties on the abundance are rather large (see Table 2), and we cannot safely assume for this star a lithium content larger than that of the other, hotter, early AGB ones.

Looking at the most luminous stars N1866\#1 and \#2, we find a totally different situation: star \#2 has an abundance definitely larger than that of the early AGB ones $(\log N(\mathrm{Li}) \gtrsim 1.5 \pm$ $0.5)$, so that we conclude that we are witnessing lithium production. No lithium line is detected in the spectra of the other star. This yields an upper limit at $\log N(\mathrm{Li})<-0.5$; on this basis we can certainly suggest that lithium in this star has been destroyed.

The amount of lithium manufactured in NGC $1866 \# 2$ is not particularly large, but because of the error size, we must wait for high dispersion spectra to set more stringent limits. The abundance is, however, in the range expected from the models (Fig. 1), also taking into account that it varies with the thermal pulse phase.

The absolute bolometric magnitude of this star is actually at the lower boundary of what we expect for the occurrence of HBB: $\left(M_{\mathrm{bol}}=-6\right)$, and that might be an additional reason for the relatively low $\log \mathrm{N}(\mathrm{Li})$ value. Figure 6 shows the Li vs. $M_{\text {bol }}$ along the same $4 M_{\odot}$ evolutionary track of Fig. 1 . The points corresponding to the observed stars are also plotted. We see that indeed the star \#2 in NGC 1866 is at the phase in which the residual lithium is completely destroyed, while the star \#1 is in a phase in which it is manufactured by HBB.

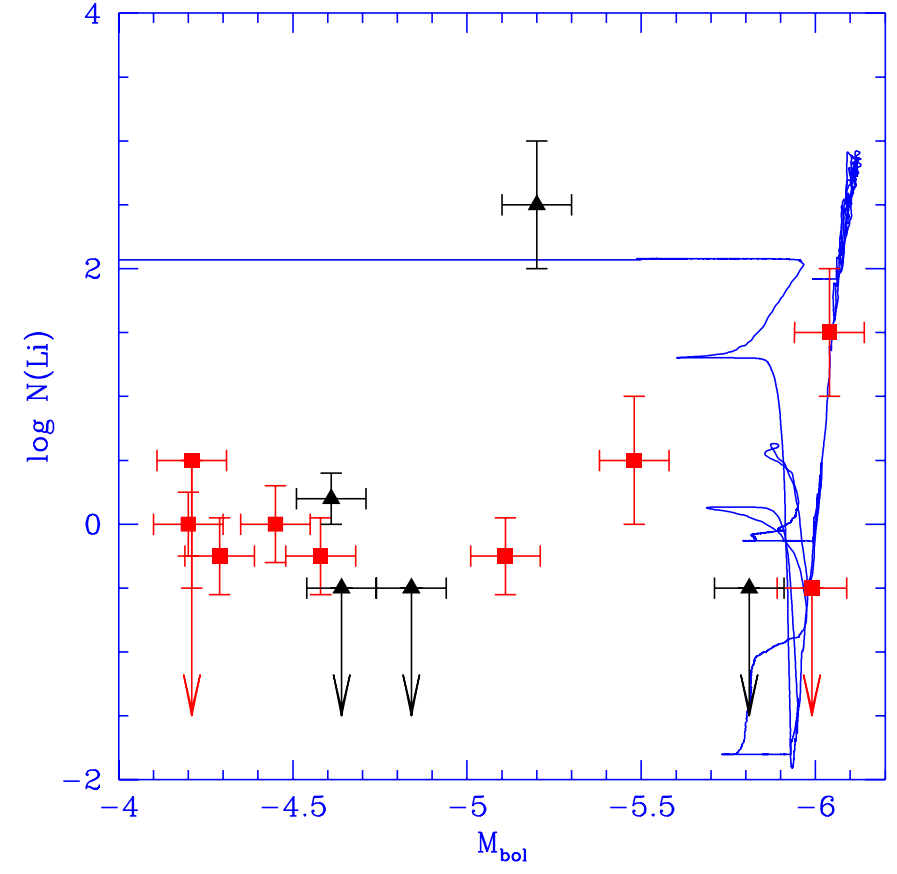

Fig. 6. Lithium abundance versus $M_{\text {bol }}$ for the target stars in NGC 1866 (squares) and NGC 2031 (triangles). The arrows represent upper limits. The evolutionary track of Fig. 1 is also shown.

The agreement with the theoretical models is quite satisfactory. Things are however less clear for the cluster NGC 2031.

\subsection{NGC 2031}

The stars in this cluster show a $\mathrm{Li}$ abundance behaviour similar to that in NGC 1866, but both the quality of some spectra and 
the fact that this cluster is much less populated than NGC 1866 make the interpretation less stringent.

First of all we have, for the stars in early AGB phase, only one reliable Li determination. This is for star (\#5), that clearly shows a lithium line of similar strength to those in the corresponding stars of NGC 1866. The abundance analysis gives $\log N(\mathrm{Li})=0.2 \pm 0.2$. Star (\#6) turned out to have a spectral type later than that expected on the basis of its photometry, a fact that casts doubts on its cluster membership. For star \#3, whose spectrum has no lithium line, we could only get an upper limit.

Concerning the later AGB objects, star \#1 seems to be very similar to star \#1 in NGC 1866: it shows no lithium (abundance $<1$ ) and it is at about the right luminosity to be in the first phase of lithium destruction due to HBB. The analysis of star \#2, whose spectra have however a relatively lower $S / N$ ratio, provides a high lithium abundance $(\sim 2.5 \pm 0.5)$ but, according to the models, this star is not luminous enough to be in the HBB phase! Mould et al. (1993) suggested, on the base their photometry and the very red $B-R(>3)$, that this star could be a very bright carbon star: in this case its large lithium abundance would imply a classification as $J$-subtype carbon star (Bouigue 1954). This possibility is ruled out, however, by the fact that its spectrum looks oxygen-rich.

\subsection{NGC 2214 and NGC 2107}

The two bottom spectra of Fig. 4 show the two stars in the clusters used as comparison terms. As shown in the figure, NGC 2214 presents a spectrum of intermediate type between the early and the later AGB stars. The abundance analysis provides $\log N(\mathrm{Li}) \simeq-0.5 \pm 0.3$. FMB90 attribute a spectral class $\mathrm{M}$ to this star, but with strong uncertainty. NGC 2107 \#1 is, on the other hand, cooler and shows clear features of a class M star (FMB90 assign an M 4 type). The TiO bands are visible but, as expected for a star with a smaller mass than predicted for lithium production, the $6707.8 \AA$ line is absent.

\section{Evolutionary stage}

Although Fig. 6 seems to provide a straightforward interpretation for the NGC 1866 lithium sequence, Fig. 1 shows that another hypothesis shall be tested, i.e. that the lithium poor star \#1 could actually be in a phase following the lithium production, when lithium is destroyed again. In this case, however, the star should have a larger bolometric luminosity and, moreover, should be surrounded by an extended circumstellar envelope, easily detectable in the mid-infrared with ISOCAM. In the ISOCAM field the three brightest sources in all bands coincide with the location of our stars \#1, \#2 and \#3, and their fluxes in mJy are given in Table 3 . The photometric results are given in Table 3, where we have as well transformed the nearIR magnitudes, previously shown in Table 2 in Jy. The fluxes in the three bands at $4.5,6.7$ and $12 \mu$ show that the most luminous source (and also reddest, if we consider the ISOCAM color [4.5]-[12]) is the star which shows high lithium abundance. It is interesting to note that the brightest star in the $K$-band (NGC $1866 \# 1$ ) is only the third in the ISO bands, and is also
Table 3. Infrared fluxes for NGC $1866^{a}$

\begin{tabular}{lccllll}
\hline \hline ID & $F_{J}$ & $F_{H}$ & $F_{K}$ & $4.5 \mu$ & $6.7 \mu$ & $12 \mu$ \\
\hline N1866\#1 & 62.5 & 99.2 & 80.4 & 6.5 & 3.7 & 1 \\
N1866\#2 & 57.5 & 89.7 & 70.7 & 8.4 & 5.0 & 2.6 \\
N1866\#3 & 40.3 & 53.0 & 49.0 & 7.2 & 4.7 & 1.7 \\
\hline
\end{tabular}

${ }^{a}$ Fluxes are in mJy.

the "bluest" in the ISO colors. This suggests a less evolved stage as an AGB star.

In general the mid-IR colors of these stars are not "extreme", indicating that they are still in the initial stages of the AGB phase. This fact almost completely discards the possibility of star NGC $1866 \# 1$ being in a later AGB phase, when the lithium is destroyed again.

Another point to examine is the star distribution along the AGB of NGC 1866. The model shown in Fig. 1 predicts a few more stars along the AGB: if we sample two stars in the first $40 \times 10^{4} \mathrm{yr}$ of evolution, we should find some other five in the following evolution, which lasts $\simeq 10^{5} \mathrm{yr}$ longer. Most of these stars could be invisible in the optical and near infrared, as they would be surrounded by a thick circumstellar envelope, but they should be the brightest objects in the field in the midIR. Actually there are no other bright mid-IR sources in the ISOCAM field, apart from the three brightest sources in the $K$-band under analysis in this paper. The absence of heavily obscured AGB stars needs to be interpreted with caution, because of the small statistics, but it is telling us that possibly the duration of this heavily obscured AGB phase is shorter than that predicted by the models, probably because of stronger than expected mass loss rate experienced by these massive AGB stars.

Although we do not have ISO data for the stars in NGC 2031, its luminous star without lithium is at about the same absolute bolometric magnitude than the similar star in NGC 1866, indicating that they probably represent a similar evolutionary phase.

What is much less obvious is the evolutionary stage of the lithium rich star in NGC 2031 at $M_{\text {bol }} \simeq-5.2$ : this is not explained by HBB models at all. Other lithium rich giants at similar magnitudes ar known, most of which are $\mathrm{J}$ stars. The so called "cool bottom processing" suggested by Wasserburg et al. (1995) could be a way to explain this anomaly. However, if the clusters NGC 1866 and NGC 2031 are indeed coeval, it is intriguing that one cluster conforms to a "standard" mixing scheme, while the other does not. It has to be noticed that, whatever the mixing process in AGB, lithium is produced through the consumption of ${ }^{3} \mathrm{He}$. If this occurs at $M_{\text {bol }} \simeq-5$, no helium is left for production at $M_{\text {bol }} \simeq-6$, where HBB becomes important. A last point to consider is that the radial velocity of NGC 2031\#2 markedly differs from those of the other stars in NGC 2031, also taking into account the large errors. As this cluster field is very crowded, it is also possible that it is a background star. 


\section{Conclusions}

1. The early AGB stars in NGC 1866 show lithium abundances which are roughly constant with increasing luminosity, consistent with the lithium dilution expected to have taken place during the previous evolutionary stages. The average abundance found is $\log N(\mathrm{Li}) \simeq 0.0 \pm 0.5$, implying, however, stronger than standard dilution. This result is confirmed by at least another early AGB star in NGC 2031, although the data derived from other stars analyzed in this cluster are inconclusive;

2. We have detected three cool luminous AGB stars in NGC 1866. The faintest one (\#3) shows a lithium abundance consistent with the remnant abundance expected from an AGB star at the beginning of this phase as a consequence of previous lithium dilution. The brightest one (\#1) does not show any lithium and the upper limit derived $(\log N(\mathrm{Li}) \leq-0.5)$ suggests that this star plausibly is in the phase of $\mathrm{HBB}$ preceding lithium production (confirmed by the smaller mid-IR excess detected by ISOCAM). The other most luminous AGB star (\#2) has a larger lithium abundance $(\log N(\mathrm{Li}) \simeq 1.5 \pm 0.5)$, which we can attribute to production by HBB. On the basis of our models we would expect a few $(\sim 5)$ other AGBs in the field of NGC 1866, while none were found, neither luminous in the optical, nor in the mid-IR. This result put interesting constraints on the duration of the AGB phase and the severity of the mass loss during this phase.

3. The most luminous star in NGC 2031 (\#1) is found to be similar to star \#1 in NGC 1866 . The second brightest star in the $K$-band in this cluster (\#2) has the largest lithium abundance in the sample, but $\mathrm{Li}$ production is not predicted at its derived luminosity (HBB not active). Further observations are needed to confirm the abundance analysis and the membership of this star.

We conclude that, though many points still remain unclear in the interpretation of the observations, we are in presence of an interesting sample of stars, whose further careful analysis can shed light on the expected evolutionary paths. Observations at a higher dispersion are needed for these stars to clarify their evolutionary status: for example, the presence of $s$-process elements in the spectra would be an important indicator of how many thermal pulses the stars have gone through. Our best guess is that they still are at the beginning of the TP phase, so that we should not expect a sensible $s$-process abundance enhancement.

Acknowledgements. CM, VT and FD acknowledge discussions with Paolo Ventura and Paolo Persi, and the support of the Italian Ministry of University, Scientific Research and Technology (MUIR) within the Cofin 1999 Project: "Stellar Dynamics and Stellar Evolution in Globular Clusters: a Challenge for the New Astronomical Instruments". PGL acknowledges support from grant
PB97-1435-C02-02 from the Spanish Dirección General de Enseñanza Superior e Investigación Científica (DGESIC).

\section{References}

Alvarez, R., \& Plez, B. 1998, A\&A, 330, 1109

Asplund, M., Gustafsson, B., Kiselman, D., \& Eriksson, K. 1997, A\&A, 318, 521

Banks, T., Dodd, R. J., \& Sullivan, D. J. 1995, MNRAS, 274, 1225

Barmina, R., Girardi, L., \& Chiosi, C. 2002, A\&A, 385, 847

Bessell, M. S., \& Brett, J. M. 1988, PASP, 100, 1134

Bessell, M. S., Castelli, F., \& Plez, B. 1998, A\&A, 333, 231, BCP98

Blöcker, T. 1995, A\&A, 297, 727

Blommaert, J., Siebenmorgen, R., Coulais, A., et al. 2002, The ISO Handbook vol. II: CAM - The ISO Camera, ESA SP-1262, SAI99-057/Dc, version 1.3

Bouigue, R. 1954, Ann. Astrophys., 7, 104

Cameron, A. G. W., \& Fowler, W. A. 1971, ApJ, 164, 111

Canuto, V. M., Goldman, I., \& Mazzitelli, I. 1996, ApJ, 473, 550

Charbonnel, C. 1995, ApJ, 453, L41

Corsi, C. E., Buonanno, R., Fusi Pecci, F., et al. 1994, MNRAS, 271, 385

Elson, R. A. W. 1991, ApJS, 76, 185

Fischer, P., Welch, D. L., Cote, P., Mateo, M., \& Madore, B. F. 1992, AJ, 103, 857

Frogel, J. A., Mould, J., \& Blanco, V. M. 1990, ApJ, 352, 96 (FMB90)

Girardi, L., Chiosi, C., Bertelli, G., \& Bressan, A. 1995, A\&A, 298, 87

Gustafsson, B., Bell, R. A., Eriksson, K., \& Nordlund, Å. 1975, A\&A, 42, 407

Gustafsson, B., Edvardsson, B., Eriksson, K., et al. 2003, in Workshop on Stellar Atmosphere Modeling, Tübingen April 2002, ed. I. Hubeny, D. Mihalas, \& K. Werner, ASP Conf. Ser., in press http://www . dstu. univ-montp2 . fr/PERSO/plez/PUBLIS/ gustafsson.ps.gz

Hill, V., Plez, B., Cayrel, R., et al. 2002, A\&A, 387, 560

Kalirai, J. S., Ventura, P., Richer, H. B., et al. 2001, AJ, 122, 3239

Kupka, F., Piskunov, N. E., Ryabchikova, T.A., Stempels, H. C., \& Weiss, W. W. 1999, A\&AS, 138, 119

Mould, J. R., Xystus, D. A., \& Da Costa, G. S. 1993, ApJ, 408, 108

Plez, B. 1998, A\&A, 337, 495

Plez, B., Brett, J. M., \& Nordlund, Å. 1992, A\&A, 256, 551

Plez, B., Smith, V. V., \& Lambert, D. L. 1993, ApJ, 418, 812

Rieke, G. H., \& Lebofsky, M. J. 1985, ApJ, 288, 618

Robertson, J. W. 1974, A\&AS, 15, 261

Sackmann, I. J., Smith, R. L., \& Despain, K. H. 1974, ApJ, 187, 555

Sackmann, I. J., \& Boothroyd A. I. 1992, ApJ, 392, L71

Smith, V. V., \& Lambert, D. L. 1989, ApJ, 345, L75

Smith, V. V., \& Lambert, D. L. 1990, ApJ, 361, L69

Smith, V. V., Plez, B., Lambert, D. L., \& Lubowich, D. A. 1995, ApJ, 441,735

Testa, V., Ferraro, F. R., Chieffi, A., et al. 1999, AJ, 118, 2839

Ventura, P., Zeppieri, A., Mazzitelli, I., \& D’Antona, F. 1998, A\&A, 334, 953

Ventura, P., D’Antona, F., \& Mazzitelli, I. 1999, ApJ, 524, L111

Ventura, P., D'Antona, F., \& Mazzitelli, I. 2000, A\&A, 363, 605

Wasserburg, G. J., Boothroyd, A. I., \& Sackmann, I.-J. 1995, ApJ, 447, L37 